\title{
Phase coherent splitting of Bose-Einstein condensates with an integrated magnetic grating
}

\author{
A. Günther, S. Kraft, C. Zimmermann, and J. Fortághฬ \\ Physikalisches Institut der Universität Tübingen, \\ Auf der Morgenstelle 14, 72076 Tübingen, Germany
}

(Dated: May 6, 2018)

\begin{abstract}
We report the phase coherent splitting of Bose-Einstein condensates by means of a phase grating produced near the surface of a micro-electronic chip. A lattice potential with a period of $4 \mu \mathrm{m}$ is generated by the superposition of static and oscillating magnetic fields. Precise control of the diffraction is achieved by controlling the currents in the integrated conductors. The interference of overlapping diffraction orders is observed after $8 \mathrm{~ms}$ of propagation in a harmonic trap and subsequent ballistic expansion of the atomic ensemble. By analyzing the interference pattern we show a reproducible phase relation between the diffraction orders with an uncertainty limited by the resolution of the diffraction grating.
\end{abstract}

PACS numbers: 03.75.Lm, 03.75.Dg, 39.20.+q

Atom optics and matter wave interferometry have made enormous progress during the last years. $\mathrm{Nu}$ merous experiments have been performed with BoseEinstein condensates serving as phase coherent sources of atomic matter waves [1]. As a part of these, diffraction from standing and moving optical lattice potentials has been demonstrated to preserve the condensate's coherence properties and is now established as a versatile tool for coherent manipulation [2, 3, 4, 5, 6, 7]. The implementation of similar diffraction scenarios on atomic micro chips is thus one of the most intriguing challenges of integrated atom optics. It holds great promise for transportable, high precision inertial force and gravity sensors [1], and for building complex atom optical circuits, e.g. quantum registers for information processing 8]. Although Bose-Einstein condensates are routinely loaded into magnetic micro traps [9], it is technically demanding to integrate atom optical elements on a chip that allows for coherent manipulation of matter waves. Recently, diffraction from optical [10] and magnetic lattices [11], as well as dynamical splitting of condensates in double well potentials [12, 13, 14] have been studied on micro chips.

In this Letter, we demonstrate phase coherent splitting of Bose-Einstein condensates by means of diffraction on an integrated magnetic grating and realize a novel interferometric scheme. The magnetic grating is generated near the surface of micro fabricated current conductors (Fig 11). We use static and oscillating magnetic fields for shaping and controlling the grating potential. Thereby, sensitive control of the populations of diffraction orders is achieved. Due to the relatively large lattice constant of $4 \mu \mathrm{m}$ and a correspondingly small recoil momentum, the diffraction orders of a condensate remain spatially overlapped during ballistic expansion. We observe their matter wave interference with a highly reproducible spatial fringe position. This provides experimental proof that diffraction from the magnetic grating preserves phase re-

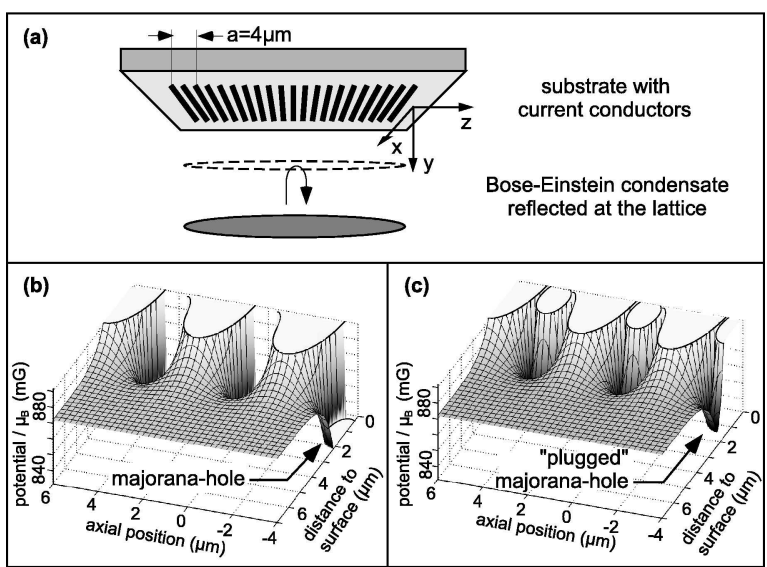

FIG. 1: (a) A condensate is scattered on an integrated magnetic grating by a controlled center of mass oscillation inside the magnetic trap. (b) Static magnetic grating: Lattice potential generated by static magnetic fields of parallel chip conductors carrying opposite currents and the field of the magnetic trap. Steep potential wells and hills alternate next to neighboring conductors. (c) Radio frequency controlled magnetic grating: Superposition of an oscillating magnetic field parallel to the lattice conductors generates adiabatic potentials at the chip surface. The plot reveals wells with limited depth and repulsive potential walls next to each conductor.

lation between the diffraction orders of a Bose-Einstein condensate. Magnetic gratings as described in this article will be important for phase coherent manipulation of matter waves in integrated atom optical circuits and for the realization of compact atom interferometers. In addition, microfabricted gratings extend the range of lattice constants accessible with optical standing wave fields [2]. In particular, the size and the structure of the unit cell can be defined by the geometry of chip conductors.

The experiment is illustrated in Fig,1a. An elongated Bose-Einstein condensates of about $1.2 \times 10^{5}{ }^{87} \mathrm{Rb}$ atoms in the $F=2, m_{F}=2$ hyperfine ground state is pre- 

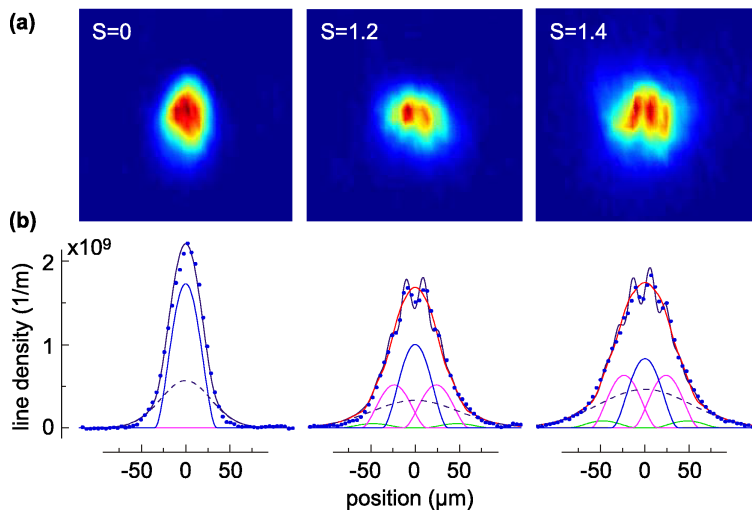

FIG. 2: (Color online) (a) Absorption images of diffracted condensates taken after $8 \mathrm{~ms}$ propagation in the magnetic trap followed by $20 \mathrm{~ms}$ of ballistic expansion. Diffraction is observed as a broadening of the cloud and by the emergence of interference fringes. The fringe contrast is limited by the optical resolution of our imaging system. (b) Vertically integrated absorption profiles of the condensate (dots). The data is fitted by the incoherent sum of diffraction orders to obtain $S$ (red line). Illustrated is also the position and the population of individual diffraction orders (solid lines) and the thermal background (dashed line). Details of the line density profiles are explained by fitting the data with a coherent superposition of the diffraction orders (blue line).

pared in a magnetic micro trap which is placed $30 \mu \mathrm{m}$ below the magnetic grating [15]. The trap is characterized by the axial and radial oscillation frequencies of $\left(\omega_{a}, \omega_{r}\right)=2 \pi \times(16,76) \mathrm{s}^{-1}$, and the offset field of $B_{z}=0.87 \mathrm{G}$ in the trap center. Diffraction of the condensate is initiated by suddenly reducing the trap-surface separation by a controlled displacement. The condensate interacts with the lattice at the upper turning point of the radial center of mass oscillation inside the trap (ydirection) followed by a swing back towards the trap center. The trap is turned off $12 \mathrm{~ms}$ after the displacement and the condensate is detected after $20 \mathrm{~ms}$ of ballistic expansion by absorption imaging. The imaging axis is parallel to the lattice conductors ( $x$-direction). Diffraction is observed as a broadening of the cloud. Additionally, the emergence of interference fringes is observed (Fig 2).

The magnetic lattice potential is generated by a set of parallel current conductors on the chip surface. The $1 \mu \mathrm{m}$ wide gold conductors are micro fabricated on a silicon substrate with a separation of $1 \mu \mathrm{m}$ between nearest neighbors [15]. Adjacent conductors carry constant currents of opposite sign: $\pm 0.2 \mathrm{~mA}$. The magnetic field of this wire configuration is superposed onto the trapping field including the offset field along $z$. The resulting magnetic field modulus has a periodicity of $4 \mu \mathrm{m}$ along $z$ and the modulation amplitude decreases exponentially from the chip surface on the length scale of the lattice constant. Steep potential wells and hills emerge close to the lattice wires (Fig 1b).
Diffraction on such a magnetic lattice potential [11] is associated with a significant loss of atoms (Fig 3). The dominant loss mechanism is due to the steep magnetic field gradients inside the potential wells of the lattice (Fig1b). Atoms entering the wells are lost due to Majorana spin flips which result from the sudden break down of the adiabatic condition along the atom's trajectory [16]. Majorana loss may be tolerable if the experiment relies on a single diffraction pulse. Numerous interferometric schemes, however, require sequences of diffraction pulses [5, 6, 7] and the loss of atoms represents a limitation.

We eliminate losses by introducing radio frequency (rf) controlled adiabatic potentials [13, 17] to "plug" the Majorana hole and observe enhanced reflectivity on the magnetic lattice. An oscillating magnetic field of $10 \mathrm{mG}$ amplitude is superposed to the static field of the lattice. The polarization is parallel to the lattice conductors and the frequency of $585 \mathrm{kHz}$ is below the Larmor frequency of $610 \mathrm{kHz}$ corresponding to the offset field $B_{z}$ in the trap center. The resulting adiabatic lattice potential features wells of limited depth and the initially steep, attractive potential slopes are converted into repulsive potential walls (Fig, 1: $)$. We characterize the reduction of Majorana losses by detecting the total number of atoms reflected from the grating. The data is plotted against the phase modulation index $S$ (Fig 3). $S$ characterizes the population of the different diffraction orders as described below; e.g. for $S \approx 1.44$, the $0 t h$ and \pm 1 st diffraction orders have about the same population. The population of diffraction orders is varied by the displacement of the trap. The rf field is activated simultaneously to the displacement and is deactivated prior to turning off the magnetic trap for ballistic expansion. The rf controlled magnetic grating displays high reflectivity compared to its static counterpart. From this result we deduce that the motion of atoms is adiabatic in the rf controlled lattice potential. The high reflectivity diffraction grating allows phase coherent manipulation of Bose-Einstein condensates and the realization of a matter wave interferometer on a chip. The observation of a deterministic phase relation between diffraction orders of the condensate is the central result of this Letter.

The interaction of the condensate with the magnetic grating is described by the model of matter wave diffraction in the Raman-Nath regime [18]. In particular, we assume a short interaction with a one-dimensional grating. This way the action of the grating is reduced to a phase modulation of the condensate wavefunction:

$$
\begin{aligned}
\Psi(z) & =\sqrt{\rho_{0}(z)} \exp \left(-\frac{i}{\hbar} \int U(z, t) \mathrm{d} t\right) \\
& =\sqrt{\rho_{0}(z)} \exp (-i S \cos (k z+\phi)) .
\end{aligned}
$$

Here, $\rho_{0}$ is the condensate's density, $U(z, t)=u(t)$. $\cos (k z+\phi)$ is the lattice potential, and the wave vector 


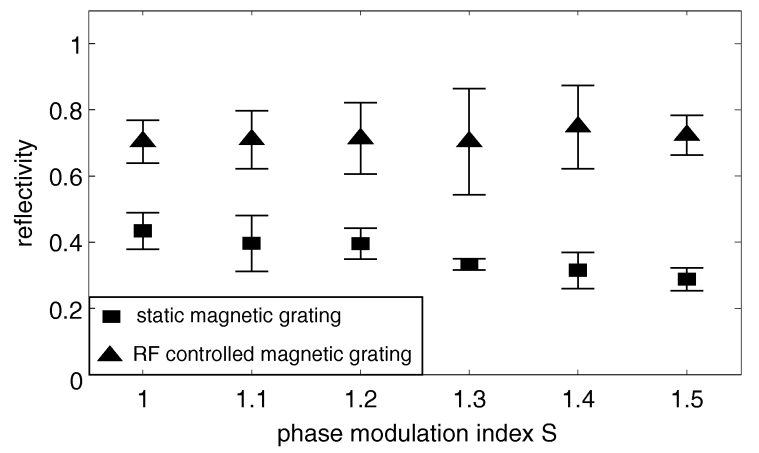

FIG. 3: Reflectivity of the static (squares) and the radio frequency controlled (triangles) magnetic grating. The data is plotted against the phase modulation index $S$. The error bars result from averaging 3-10 individual measurements (standard deviation). $S$ is fitted with an accuracy of about 0.1 .

$k=2 \pi / a$ is given by the lattice constant $a . \phi$ characterizes a shift of the lattice position with respect to the center of the condensate. The amplitude $u(t)$ accounts for the varying modulation depth during reflection as seen by the atom along its trajectory. The accumulated phase can be summarized using the phase modulation index $S$.

The validity of this approximation is verified by numerical solution of the three-dimensional Gross-Pitaevskii equation [19]. It has been shown that the RamanNath approximation holds for $E_{r} \tau \ll \hbar / S$, where $E_{r}=$ $(\hbar k)^{2} / 2 m$ is the recoil energy and $\tau$ the effective interaction time with the lattice [18]. With $\tau<0.2 m s$ this condition is well satisfied in our experiment.

The wavefunction (Eq 2) can be expanded into momentum eigenfunctions of the axial motion:

$$
\Psi(z)=\sqrt{\rho_{0}(z)} \sum_{n=-\infty}^{\infty} e^{i n(\phi-\pi / 2)} J_{n}(S) e^{i n k z} .
$$

Here, $J_{n}$ are Bessel functions of first kind and $n$ is an integer. The total wavefunction $\Psi=\sum \Psi_{n}$ is therefore a superposition of the discrete momentum eigenfunctions $\Psi_{n}$, describing the wavefunction of the $n^{\text {th }}$ diffraction order. The probability for an atom to be diffracted into the $n^{\text {th }}$ order is proportional to $\left|J_{n}(S)\right|^{2}$, and the relative phase between neighboring orders is $(\phi-\pi / 2)$.

By exploiting the dependence of $S$ on the potential modulation (Eq1 and 22) we control the population of the diffraction orders with the radio frequency field. The frequency and the amplitude of the oscillating field determines the coupling between internal atomic states and with it the depth and shape of the potential wells. Radio frequency control of diffraction (Fig (4) may be particulary convenient for integrated atom-optical devices when sequences of diffraction pulses are required. The phase modulation index can be electronically changed while the condensate performs a constant center of mass oscillation in the trap and interacts with the grating in subsequent oscillation periods.
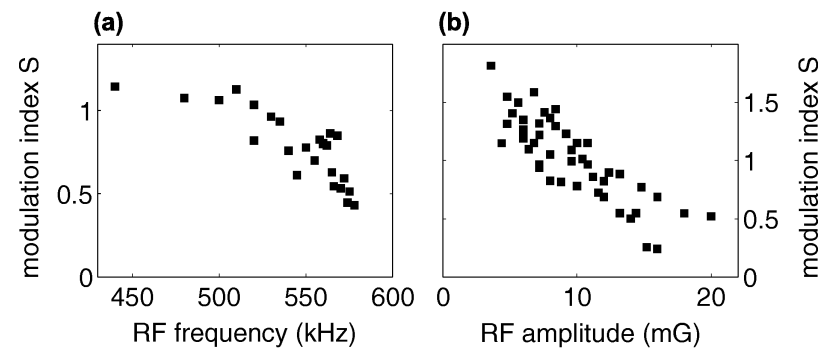

FIG. 4: Control of diffraction by means of the oscillating magnetic field. The diffraction is initiated by a displacement $d$ of the trap center towards the grating. (a) At constant amplitude of $10 \mathrm{mG}$ the frequency is used to control the diffraction. $d=14 \mu \mathrm{m}$. (b) At constant frequency of $585 \mathrm{kHz}$ the amplitude is used to control the diffraction. $d=14.5 \mu \mathrm{m}$.

The experimental results are derived from absorption images as illustrated in Fig, 2, For the analysis, the images are summed up along the y-coordinate, perpendicular to the course of diffraction. The resulting line density profile is fitted first by the incoherent superposition of the diffraction orders, $\rho_{\text {incoh }}=\sum\left|\Psi_{n}\right|^{2}$, to obtain the phase modulation index $S$. Here, we assume that, at the time of absorption imaging, the centers of neighboring diffraction orders are separated by a distance of $\Delta z=\int \hbar k(t) / m \mathrm{~d} t \approx 24 \mu \mathrm{m}$ due to $8 \mathrm{~ms}$ propagation in the trap followed by $20 \mathrm{~ms}$ ballistic expansion [20]. The repulsive atomic mean-field interaction is included by assuming the usual ballistic expansion of condensates [21]. Thermal atoms are taken into account by a Gaussian contribution to the line density profile. The incoherent sum of diffraction orders fits well to the overall cloud envelope but it does not explain further details of the cloud.

Unlike diffraction from standard optical lattice potentials [2, 3, 4, 4, 5, ㅎ, 7, 10], the recoil energy $E_{r}=(\hbar k)^{2} / 2 m$ transferred by the $4 \mu \mathrm{m}$ lattice is smaller than the chemical potential of the condensate. The diffraction orders do not separate entirely during ballistic expansion and the spatial distribution of the condensate is given by the coherent superposition of the diffraction orders [22]: $\rho_{\text {coh }}=\left|\sum \Psi_{n}\right|^{2}$. We observe high contrast interference fringes of overlapping diffraction orders for phase modulation indices between $1.1-1.6$ (Fig 21). The fringes are dominated by the interference of the $0 t h$ and \pm 1 st orders with populations that are comparable in this range.

The phase of the individual diffraction orders after ballistic expansion results from evaluating the action integral [18]. It contains various contributions: the parabolic phase profile characterized by a curvature $\alpha$ [21], the center of mass momentum of the diffraction order given by $k$ [5], and phase contributions $\phi_{\text {trap }}$ and $\phi_{\text {tof }}$ accumulated during propagation in the trap and during time of flight. Interference of overlapping diffraction orders results from 
(a)

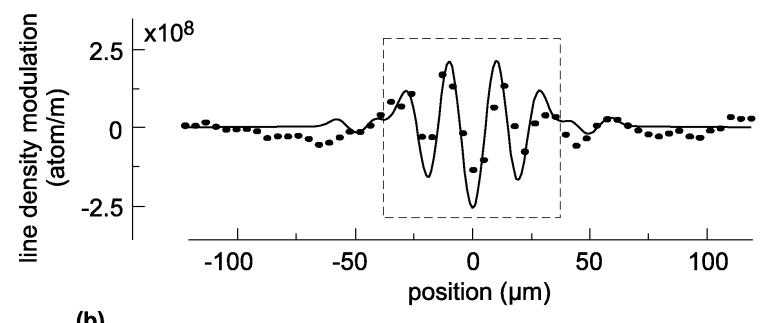

(b)

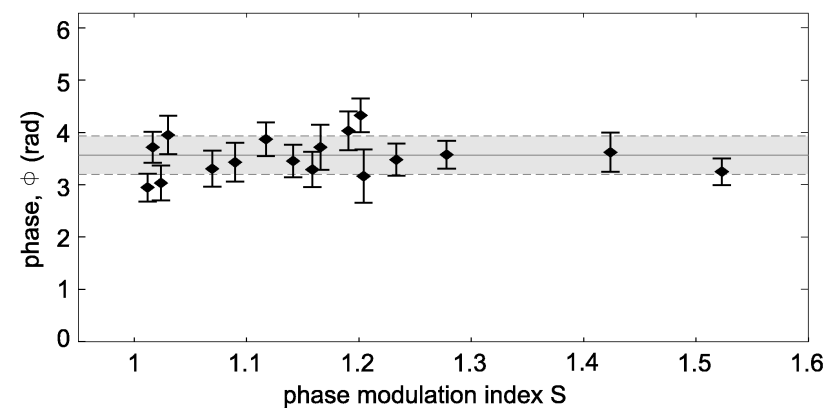

FIG. 5: Interference of overlapping diffraction orders. High contrast interference fringes are observed for $S=1.1-1.6$. (a) Line density modulation of the cloud (dots) for $\mathrm{S}=1.2$. The solid line is the fit by the coherent superposition of the diffraction orders. (b) The phase $\phi$ is derived from the fit of the center part of the line density modulation. Each data point is a single measurement, the error bars indicate the standard deviation of each fit. The average phase spread is $\pi / 8$.

the coherent superposition of the wavefunctions

$$
\begin{aligned}
\Psi_{n}(z)= & \sqrt{\rho(z-n \Delta z)} J_{n}(S) \\
& \cdot e^{i\left[\alpha(z-n \Delta z)^{2}+n(\phi-\pi / 2)+n k(z-n \Delta z)+\phi_{\mathrm{trap}, n}+\phi_{\mathrm{tof}, n}\right]}
\end{aligned}
$$

We restrict our analysis to the reproducibility of the interference fringes for different realizations of the experiment. A reproducible spatial density distribution of atoms is a direct signature of phase coherent splitting and manipulation of the condensate 12,23$]$. The reproducibility of the interference fringes relies on the reproducibility of the spatial position of the condensate relative to the lattice. A variation of the position would show up as a variation of $\phi$. Other phase contributions are set by the experimental conditions and do not change between successive realizations of the experiment. By fitting the data with the coherent superposition of the diffraction orders varying only the single parameter $\phi$, we find that the relative phase of the diffraction orders is deterministic with an uncertainty of $\pi / 8$ on average (standard deviation). A typical interference pattern is illustrated in (Fig $5 \mathrm{~b})$. It shows the line density modulation of the cloud: $\rho_{\mathrm{coh}}-\rho_{\text {incoh }}$. The phase $\phi$, derived from the central part of the interference pattern, is plotted in Fig 5 5 . Taking into account the condensate's axial size of $(\sim 50 \mu \mathrm{m})$ and the number of "illuminated" lattice sites $(\sim 12)$ the observed phase spread is "diffraction limited", i.e. given by the resolution of the lattice $\Delta k / k=1 / n$.
This uncertainty due to the finite size of the condensate could be further reduced by expanding the axial size of the cloud.

From the highly reproducible interference fringes we conclude that splitting of condensates with an integrated magnetic grating is phase coherent. In addition, we have proven that the experimental parameters can be controlled with an accuracy sufficient for the construction of integrated matter wave interferometers. In contrast to other schemes, the interferometer presented here requires a single diffraction event only. The interferometric path is closed by the ballistic expansion due to mean-field repulsion of the condensate atoms. For above experimental conditions, the resolution of a force detector is on the order of $10^{-4} \mathrm{~g}$. The simultaneous interference of more than two diffraction orders allows not only for the detection of forces but also force gradients, and even higher spatial derivatives of external forces.

We thank C. J. Vale, T. Judd, and M. Fromhold for helpful comments on the manuscript. Financial support from the Landesstiftung Baden-Württemberg, and the European Union (MRTN-CT-2003-505032) is greatly acknowledged.

* Electronic address: fortagh@uni-tuebingen.de

[1] M. A. Kasevich, Science 298, 1363 (2002).

[2] E. W. Hagley et al., Science 283, 1706 (1999).

[3] J. Stenger et al., Phys. Rev. Lett. 82, 4569 (1999).

[4] M. Kozuma et al., Phys. Rev. Lett. 82, 871 (1999).

[5] J. E. Simsarian et al., Phys. Rev. Lett. 85, 2040 (2000).

[6] K. Bongs et al., Phys. Rev. A 63, 031602(R) (2001).

[7] Y. Torii et al., Phys. Rev. A 61, 041602(R) (2000).

[8] G. Birkl and J. Fortágh, Las. Phot. Rev. 1, 12-23 (2007).

[9] J. Fortágh and C. Zimmermann, Rev. Mod. Phys. 79, 235 (2007).

[10] Y. Wang et al., Phys. Rev. Lett. 94, 090405 (2005).

[11] A. Günther et al., Phys. Rev. Lett. 95, 170405 (2005).

[12] Y. Shin et al., Phys. Rev. A 72, 021604(R) (2005).

[13] T. Schumm et al., Nature Physics 1, 57 (2005).

[14] G.-B. Jo et al., Phys. Rev. Lett. 98, 030407 (2007).

[15] A. Günther et al., Phys. Rev. A 71, 063619 (2005).

[16] C. V. Sukumar and D. M. Brink, Phys. Rev. A 56, 2451 (1997).

[17] I. Lesanovsky et al., Phys. Rev. A 74, 033619 (2006), and references therein.

[18] C. Henkel, J.-Y. Courtois, and A. Aspect, J. Phys. II France 4, 1955 (1994), and references therein.

[19] T. E. Judd et al., in preparation.

[20] After displacement, the cloud is held for $12 \mathrm{~ms}$ in the trap. In the first $4 \mathrm{~ms}$ the condensate reachs the magnetic lattice and is reflected. In the following $8 \mathrm{~ms}$ the diffraction orders propagate in the axial potential of the trap.

[21] Y. Castin and R. Dum, Phys. Rev. Lett. 77, 5315 (1996).

[22] The thermal component does not contribute to the interference. Diffraction experiments with a thermal cloud just above the transition temperature for Bose-Einsten condensation do not show interference. 
[23] Y. Shin et al., Phys. Rev. Lett. 92, 050405 (2004). 